\title{
In-vivo distortion of through-plane flow by spiral phase-contrast imaging
}

\author{
lain T Pierce ${ }^{1,2^{*}}$, Peter D Gatehouse ${ }^{1,2}$, David N Firmin ${ }^{1,2}$ \\ From 15th Annual SCMR Scientific Sessions \\ Orlando, FL, USA. 2-5 February 2012
}

\section{Summary}

To explain previously unrecognised consequences of two sources of phase curvature over the vessel cross-section in spiral imaging i.e. off-resonance and the velocityencoded phase-shift.

\section{Background}

The effects of off-resonance frequency errors during spiral readouts [Yudilevich and Stark, 1987] are known. Here we explain previously unrecognised intra-voxel dephasing consequences of two sources of phase curvature over the vessel cross-section in through-plane flow imaging using spirals i.e. off-resonance and the velocityencoded phase-shift, including the consequences for invivo measurements.

\section{Methods}

Laminar through-plane flow phantom $(50 \mathrm{~cm} / \mathrm{s})$ and popliteal artery studies were acquired at Venc $=50 \mathrm{~cm} / \mathrm{s}$ with initial off-resonance offsets $\pm 010,20,40 \mathrm{~Hz}$ representing $<1 \mathrm{ppm}$ at $1.5 \mathrm{~T}$. Reference ("Ref") and velocityencoded ("Vel") magnitude and phase images were obtained (as a cine in-vivo). Spiral FOV was $150 \mathrm{~mm}$, $1 \mathrm{~mm}$ resolution, duration $25.7 \mathrm{~ms}$; TE/TR $4.0 / 32.7 \mathrm{~ms}$, FA $30^{\circ}, 4$ interleaves.

\section{Results}

Figure 1 shows magnitude and phase images for Ref and Vel scans with corresponding velocity maps (VM). For low flow rates the off-resonance blurring at $+40 \mathrm{~Hz}$ and $-40 \mathrm{~Hz}$ is similar for both Ref. and Vel. images. However, high velocities cause an apparent "implosion" (Figure 1I) and "explosion" (Figure 1-E) of the vessel for opposite off-resonance frequencies. From the distorted VMs, peak velocity was measured at $37.8,48.6,56.3 \mathrm{~cm} / \mathrm{s}$ for $-40,0,40 \mathrm{~Hz}$ off-resonance respectively.

For theoretical explanation, Figure 2a) depicts the ideal diametrical phase line profile across a tube velocity-compensated spiral imaging, red line = on-resonance and blue/green lines = phase curvature induced by $\pm 40 \mathrm{~Hz}$ off-resonance error. Figure 2b)-red shows velocity-encoded phase over the vessel with parabolic flow. Figure $2 \mathrm{~b}$ )-blue shows the consequence of their addition: the increased radial slope worsens intra-voxel dephasing in all but the central pixels of a laminar flow ("implosion" effect, Figure 1-I). In Figure 2b)-green the opposing phase curvatures lead to a cancellation of the radial phase slope at some radius, which can lie outside the true lumen ("explosion" effect, Figure 1-E).

\section{Conclusions}

The distortion of the velocity distribution over the invivo vessel distorts peak velocity by $\sim 20 \%$ at $-40 \mathrm{~Hz}$ offresonance at these sequence parameters. Separate tests eliminated through-plane gradient fields as a cause, including eddy-current effects after the velocity-encoding pulses.

These effects on magnitude images and velocity distributions at $<1 \mathrm{ppm}$ off-resonance are potentially difficult for $>20 \mathrm{~ms}$ spiral readouts in small vessel applications at least, perhaps more so near B0-distortions such as lungs. Shorter spirals and avoiding large intra-voxel radial phase shear are some-what incompatible with rapid flow work.

\section{Author details \\ ${ }^{1} \mathrm{NHLI}$, Imperial College London, London, UK. ${ }^{2} \mathrm{CMR}$ Unit, Royal Brompton Hospital, London, UK.}

Published: 1 February 2012

${ }^{1} \mathrm{NHLI}$, Imperial College London, London, UK

Full list of author information is available at the end of the article

(c) 2012 Pierce et al; licensee BioMed Central Ltd. This is an open access article distributed under the terms of the Creative Commons Attribution License (http://creativecommons.org/licenses/by/2.0), which permits unrestricted use, distribution, and reproduction in any medium, provided the original work is properly cited. 

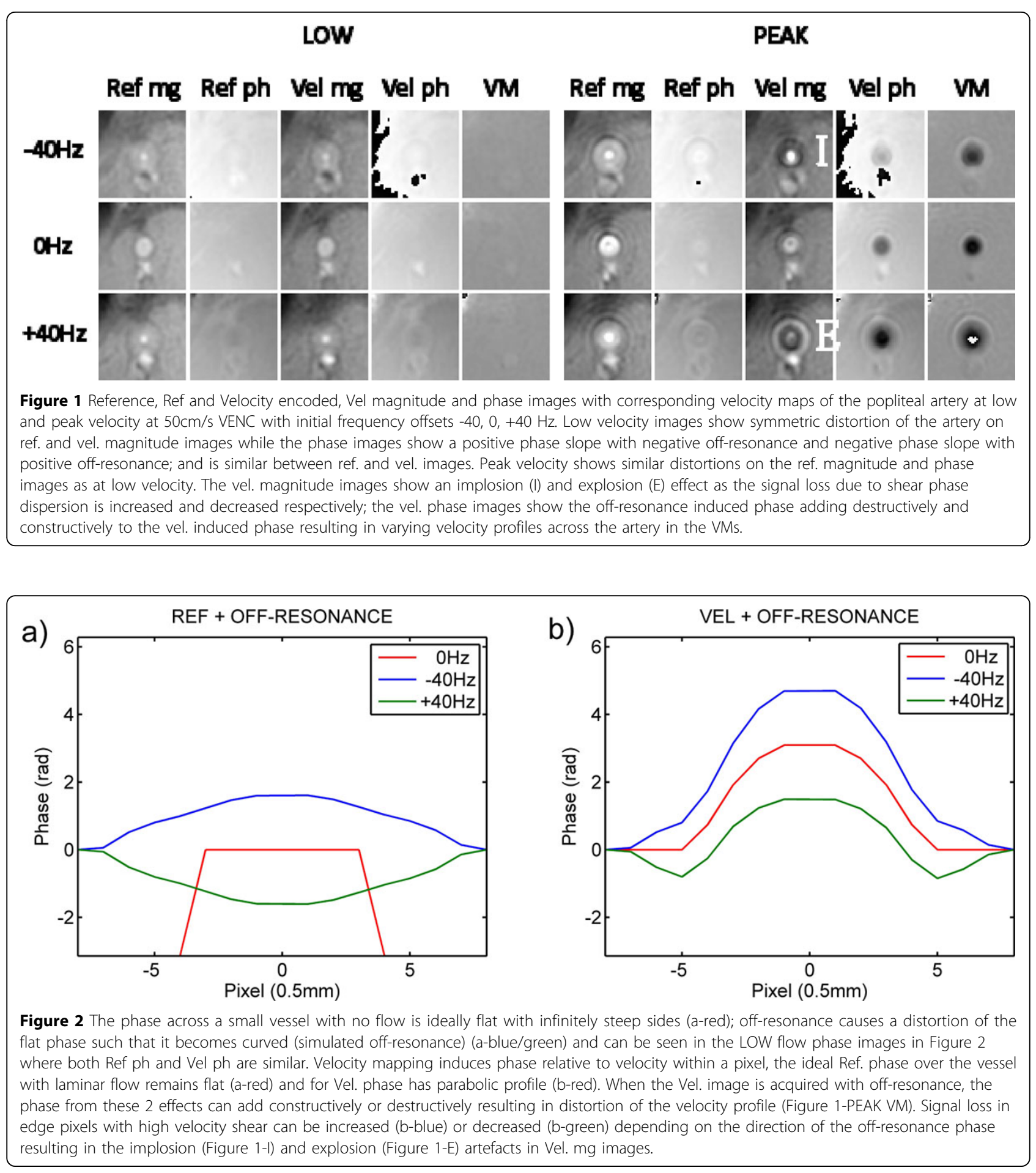

doi:10.1186/1532-429X-14-S1-W61

Cite this article as: Pierce et al:: In-vivo distortion of through-plane flow by spiral phase-contrast imaging. Journal of Cardiovascular Magnetic Resonance 2012 14(Suppl 1):W61. 